\title{
Atmospheric | Metabolic: Subsistence in the Antarctic
}

\author{
ELLEN GARRETT \\ City College of New York
}

Antarctica remains as the last bastion of vestal earth; still the perceivably vacant landscape seems an unlikely setting for the discussion of architecture. The vast continent boasts no permanent human residents and as a result has proven to be the most optimal case study of climate change. As its namesake suggests, the Anthropocene has known more widespread urban coverage than any other period. Not just urban sprawl, but all landscapes in service of such urbanism, such as industrial farming and oceanic gyres impact life at the poles. To understand humanity's global influence on nature, we glean the most substantive information from areas that thrive in our absence. Evaluating the effects on marine life, the formation and degradation of ice, and the altered conditions of the ecology will help to inform ways in which architecture can better adapt to its polar environment.

In the most remote part of the planet, with six months of darkness and complete isolation, human settlement within the Antarctic is entirely reliant on resources found elsewhere. The remoteness of the continent and the dangers of traveling in winter months prohibits a steady stream of resources, which are used cautiously. There are no readily available building materials or means by which to sustain life long term in Antarctica. Research facilities depend on continually advancing building and material technologies in order to withstand Antarctic conditions.

In response to the prompt, "Architecture of Attunement and Planetary Ecology," Atmospheric | Metabolic addresses opportunities for architecture to better adapt to a landscape that actively rejects it. Can these facilities that so closely monitor the landscape form a symbiotic relationship with its surroundings in order to evolve with the rapidly altering environment? Sean Lally speaks of expanding the capabilities of microclimates and ecosystems so as to make them architectural materials themselves. These investigations can lead us to a better understanding of the role of architecture in an increasingly volatile landscape, at the regional polar scale as well as globally. This is not to suggest that designers interfere with otherwise pristine landscapes, but instead consider how the built environment at large can be reconsidered within the context of more precarious surroundings.

While the surface of land mass in Antarctica is mostly barren and desolate, the ice shelves that extend into the Southern Ocean are teeming with life. Algae flourishes off the coast in summer months with nutrients upwelling from the ocean floor and 24 hours of sunlight. This is a major food source and an integral part of the ecosystem. Algae grows in abundance and is one of few photosynthesizing organisms on the continent. By incorporating algae into the built environment, there is an opportunity to provide significant reduction of external resources. With self-sufficiency comes reduced carbon footprint in an extremely fragile environment. The architecture in Antarctica has an opportunity to materialize energies so as to be an active testing ground that is both adaptive and informative, while helping to mitigate fallout from the build environment. 

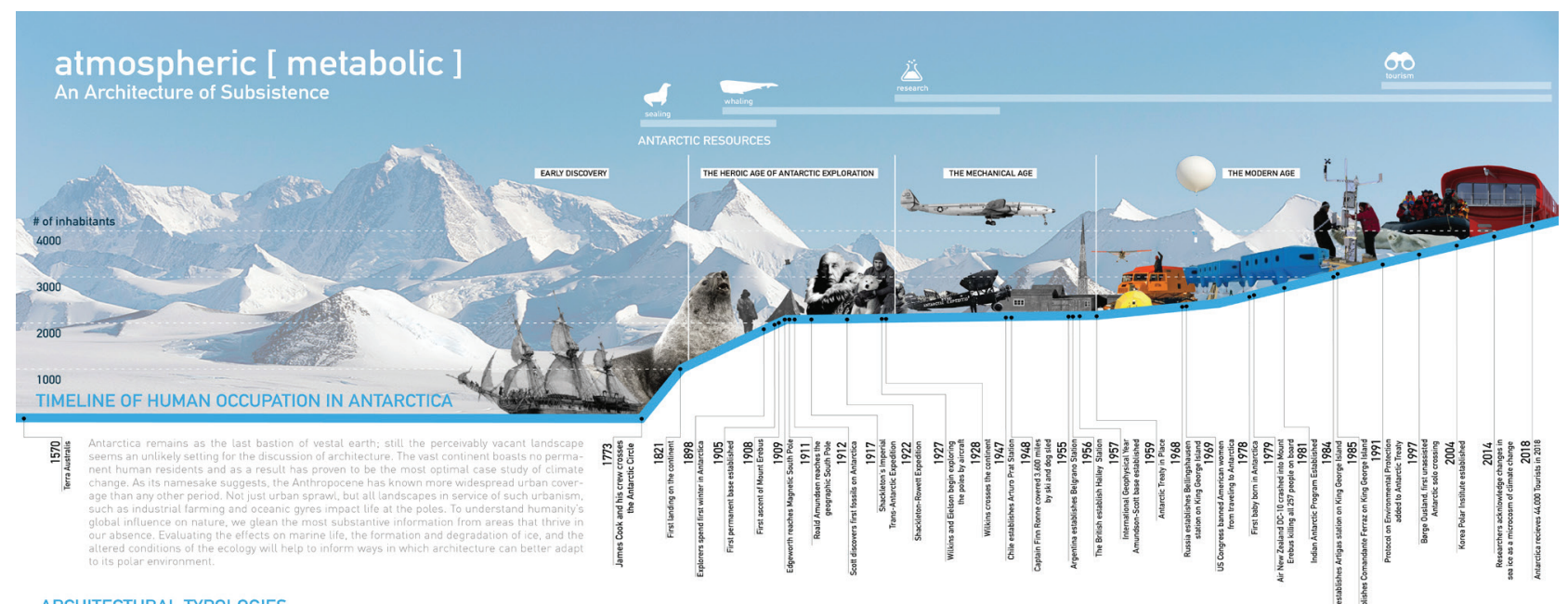
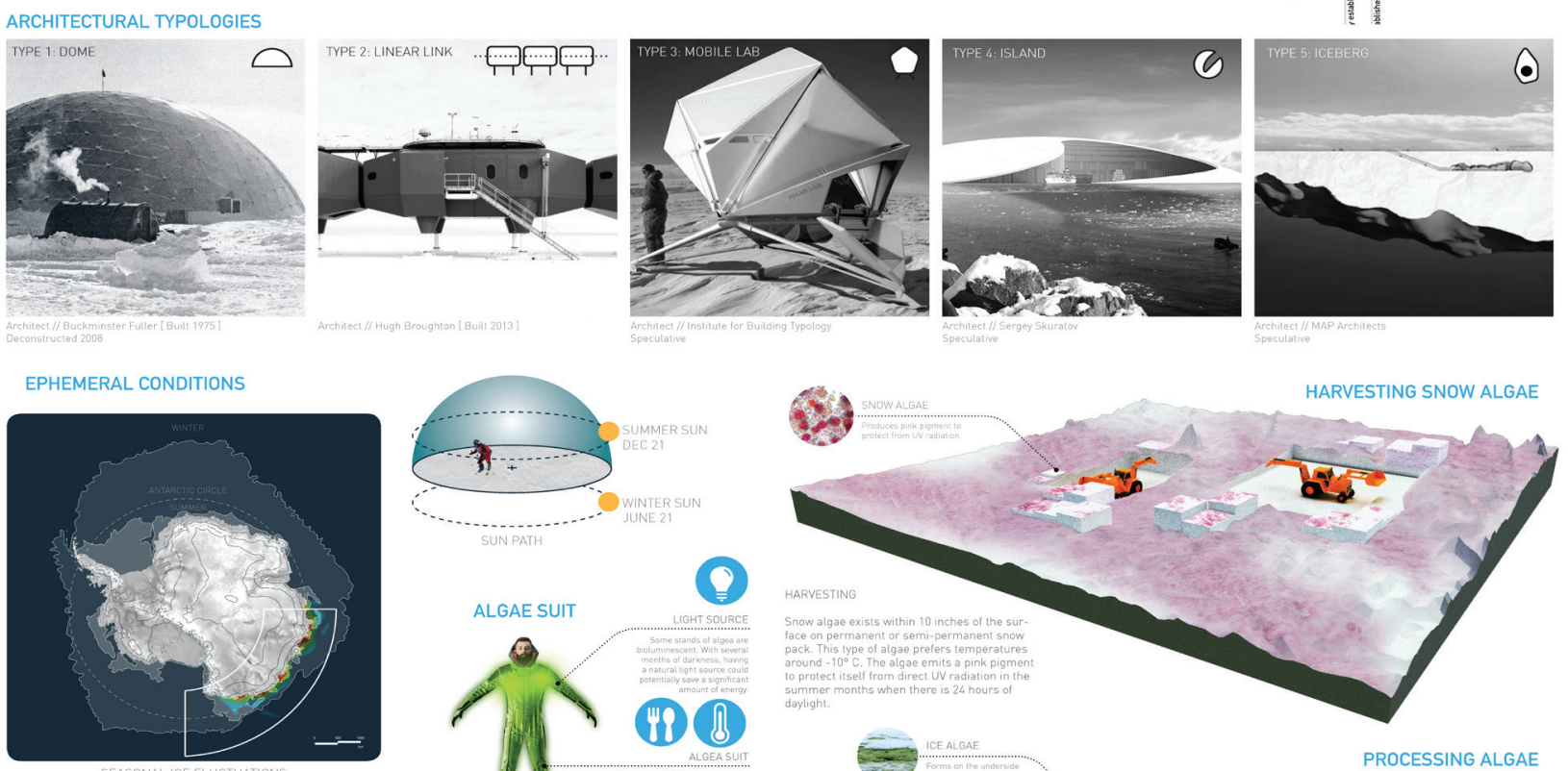

(d)
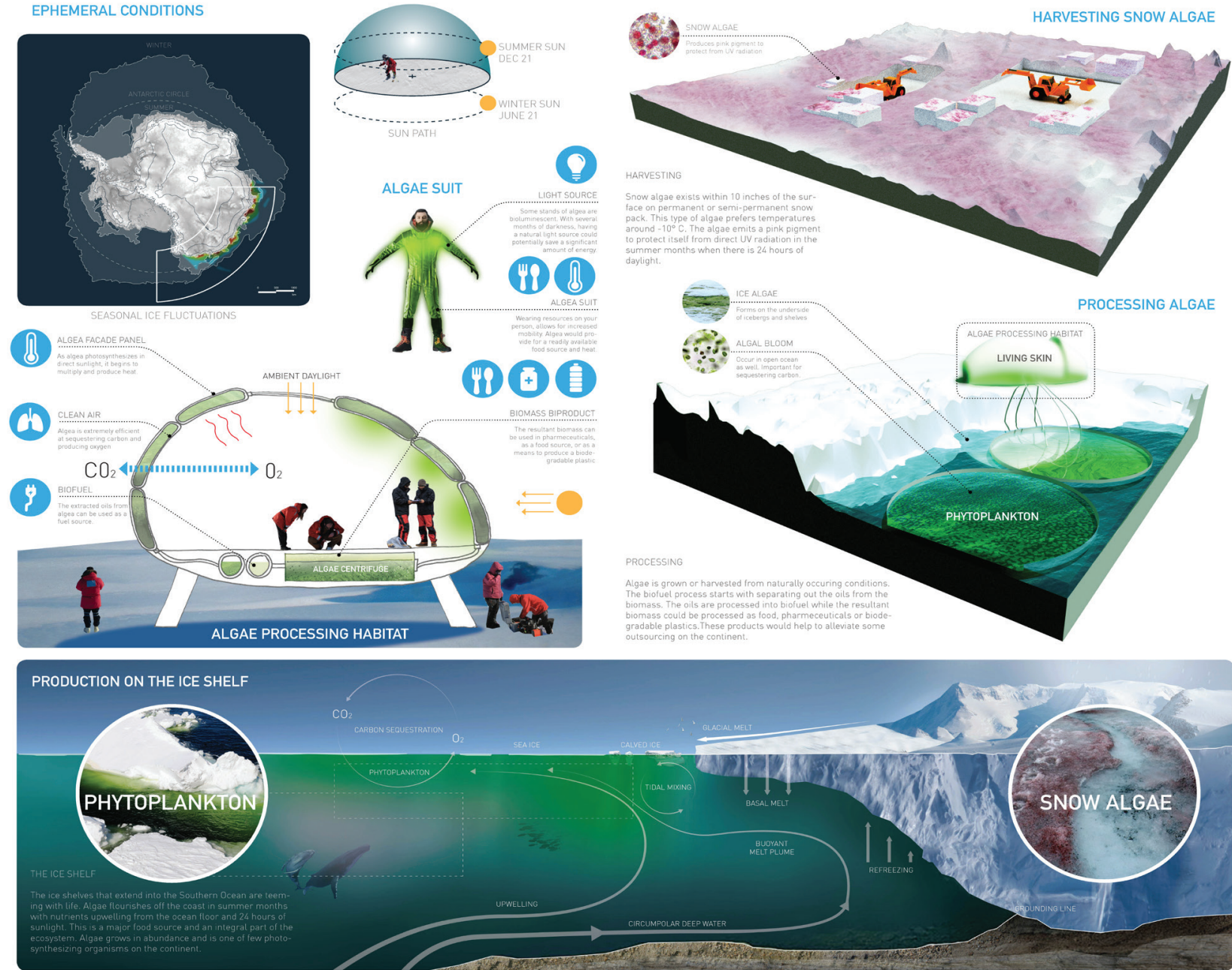